\title{
Intermixing in strained InGaAs/GaAs quantum-well infrared photodetectors
}

\author{
Alex S. W. Lee and E. Herbert $\mathrm{Li}^{\mathrm{a})}$ \\ Department of Electrical and Electronic Engineering, University of Hong Kong, Hong Kong \\ Gamani Karunasiri \\ Department of Electrical Engineering, National University of Singapore, Singapore 119260
}

(Received 4 September 1998; accepted for publication 17 December 1998)

\begin{abstract}
The effect of interdiffusion on strained InGaAs/GaAs quantum-well infrared photodetectors is investigated. Photoluminescence measurements of the interband transition indicate that there is minimal deterioration of the annealed heterostructures, as it is also evident from both the transverse electric and transverse magnetic infrared intersubband optical transitions. The absorption peak wavelength is redshifted from the as-grown $10.2 \mu \mathrm{m}$ to 10.5 and $11.2 \mu \mathrm{m}$ for 5 and $10 \mathrm{~s}$ annealing, respectively, at $850^{\circ} \mathrm{C}$ without appreciable degradation in absorption strength. The peak responsivity of the as-grown and annealed spectra is of comparable amplitude, whereas the annealed spectra become narrower in shape. The dark current of the annealed devices is about an order of magnitude higher than the as-grown one at $77 \mathrm{~K}$. (C) 1999 American Institute of Physics. [S0003-6951(99)02508-5]
\end{abstract}

Long-wavelength quantum-well infrared photodetectors (QWIPs) based on intersubband transitions (IT) have been quite well studied and are progressing rapidly. ${ }^{1}$ With the development of quantum-well (QW) growth technology and band-gap engineering, high-quality pseudomorphic strainedlayer QWs can be achieved. It has been demonstrated that normal incident strained InGaAs/GaAs QWIPs (Ref. 2) without grating coupling is possible. Recently, the thermal stability of strained QW layers subjected to heat treatment has been of great interest in the thermal diffusion-induced QW intermixing technique, which can be used to produce tunable operation wavelength optoelectronic devices, ${ }^{3}$ especially for structures with higher In concentration. Using this technology, the postgrowth tuning of the AlGaAs/GaAs intersubband absorption peak position ${ }^{4}$ and a tunable operation wavelength QWIP (Ref. 5) have been demonstrated. However, most of the studies have centered on the strain-free $\mathrm{AlGaAs} / \mathrm{GaAs}$ material system, in which the polarization selection rule requires a nonzero incident light electric-field component along the growth axis. Normal incidence is possible only with an additional optical coupling scheme that increases the complexity of device integration, such as array fabrication. Postgrowth wavelength tuning of a normal incident QWIP without surface grating has yet to be realized. In this letter, we report a wavelength tunable, grating-free, and highly strained normal incident $\mathrm{In}_{0.3} \mathrm{Ga}_{0.7} \mathrm{As} / \mathrm{GaAs}$ QWIP. We demonstrate the postgrowth tunability of the IT absorption peak wavelengths $(\Delta \lambda \approx 1 \mu \mathrm{m})$. Both the transverse electric (TE) and transverse magnetic (TM) infrared ITs are observed after interdiffusion, indicating that interdiffusion preserves the intrinsic optical characteristic of the strained QWIP material. With the redshift of the detection wavelength, the responsivity is shown to be comparable to the as-grown one by means of QW intermixing.

A multiple quantum well (MQW) was grown by molecular beam epitaxy on a (100) semi-insulating substrate. Its

a)Electronic mail: ehli@eee.hku.hk structure consists of 50 periods of a $40 \AA \mathrm{In}_{0.3} \mathrm{Ga}_{0.7} \mathrm{As}$ well and a $300 \AA \mathrm{GaAs}$ barrier. The QW structure was sandwiched between an $n^{+}$buffer $(1 \mu \mathrm{m})$ and a cap layer $(0.5$ $\mu \mathrm{m})$ and the wells were doped with $\mathrm{Si}$ at a density of about $2 \times 10^{18} \mathrm{~cm}^{-3}$. Each QW was designed to have only one bound state in the well and the first excited state in the continuum above the barrier. The energy difference between the first two eigenstates corresponds to wavelength $\cong 10 \mu \mathrm{m}$ $(\hbar \omega=124 \mathrm{meV})$. Two samples were capped with an approximately $250 \mathrm{~nm}$ thick electron-beam evaporated $\mathrm{SiO}_{2}$ dielectric layer. Rapid thermal annealing (RTA) was carried out in a halogen lamp annealing system (AST SHS10) with a double-strip graphite heater under flowing nitrogen ambient. Two samples were annealed at $850^{\circ} \mathrm{C}$ for RTA time $t_{A}=5$ and $10 \mathrm{~s}$, respectively. Mesa diodes $\left(200 \times 200 \mu \mathrm{m}^{2}\right)$ were fabricated by a standard lithography technique and wet chemical etching.

Photoluminescence (PL) measurement was performed at $4.5 \mathrm{~K}$ using $514.5 \mathrm{~nm}$ argon laser excitation with a power of $20 \mathrm{~W} / \mathrm{cm}^{2}$. Figure 1 shows the PL spectra of the as-grown and the interdiffused MQW. The PL peak shifts progressively to higher energy with the annealed time from as-

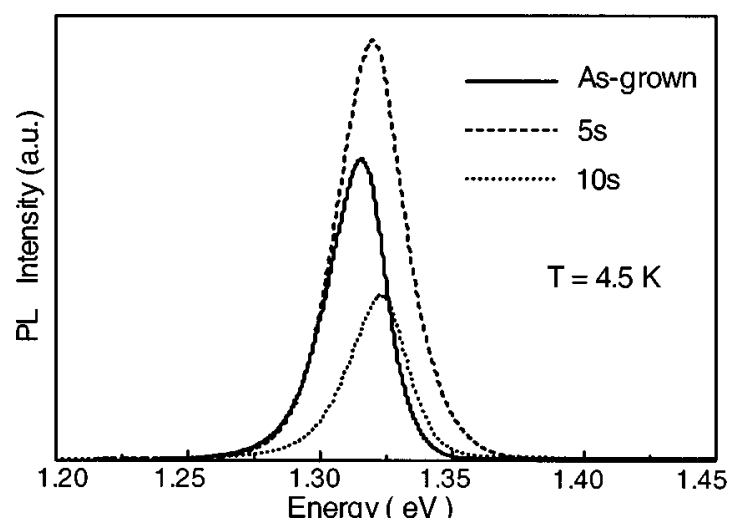

FIG. 1. Photoluminescence spectra of the as-grown, $5 \mathrm{~s}$, and $10 \mathrm{~s}$ interdiffused $\mathrm{InGaAs} / \mathrm{GaAs} \mathrm{MQW}$ at $T=4.5 \mathrm{~K}$. 

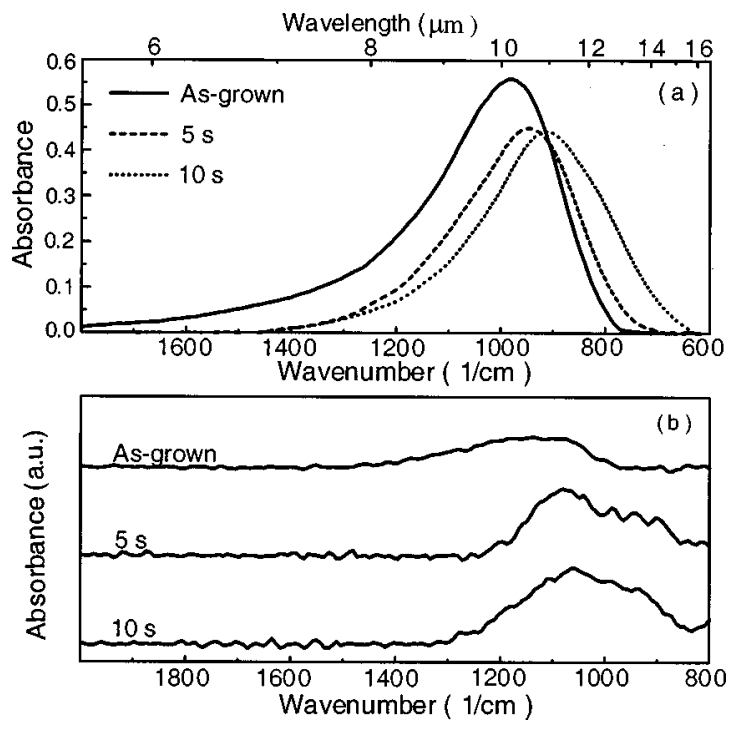

FIG. 2. Absorption spectra of the as-grown, $5 \mathrm{~s}$, and $10 \mathrm{~s}$ annealed samples at $300 \mathrm{~K}$ as a function of the wave number for (a) $0^{\circ}$ (TE+TM) polarization, and (b) $90^{\circ}$ (TE) polarization. (The curves have been shifted vertically for clarity.)

grown $1.316 \mathrm{eV}$ to 1.319 and $1.323 \mathrm{eV}$, respectively. The blueshift of the band-gap energy indicates the intermixing of group III elements near the heterointerfaces. The PL intensity is increased by nearly onefold in magnitude for the $t_{A}$ $=5 \mathrm{~s}$ sample, and decreased by almost the same order for the $t_{A}=10 \mathrm{~s}$ sample, in comparison with the as-grown intensity. The full width at half maximum (FWHM) PL linewidth does not increase much as compared to the as-grown sample, about 4 and $1 \mathrm{meV}$, for the 5 and $10 \mathrm{~s}$ annealed samples, respectively. Although the well width is below the critical thickness for 30\% In concentration, a small amount of dislocation can still exist in highly strain QWs due to a partial relief of strain. ${ }^{6,7}$ The small variation in PL intensity and FWHM at current experimental conditions may suggest that the onset of dislocation generation has not been initiated and that the structural quality is preserved. ${ }^{7}$

The intersubband absorption measurement was measured using a Nicolet Magna-IR 850 Fourier transform IR spectrometer at room temperature with a $45^{\circ}$ polished multipass waveguide geometry. The effect of interdiffusion on the optical absorption of annealed QWs is evident in Fig. 2, where the as-grown absorption peak is redshifted continuously for both polarizations. Figure 2(a) shows that, at $0^{\circ}$ polarization, the absorption peak is shifted from the asgrown $980 \mathrm{~cm}^{-1}$ (at $10.2 \mu \mathrm{m}$ ) to 952 (at $10.5 \mu \mathrm{m}$ ) and $893 \mathrm{~cm}^{-1}$ (at $11.2 \mu \mathrm{m}$ ), for the $t_{A}=5$ and $10 \mathrm{~s}$ samples, respectively. The normal incident (TE) absorption, which is the result of band-mixing effects induced by the coupling between the conduction and valence bands, ${ }^{8}$ is preserved after interdiffusion, as shown in Fig. 2(b). As seen from Fig. 2(b), the absorption peak is redshifted continuously with increasing $t_{A}$ from $1124 \mathrm{~cm}^{-1}$ (at $8.9 \mu \mathrm{m}$ ) to 1080 (at 9.26 $\mu \mathrm{m})$ and $1057 \mathrm{~cm}^{-1}$ (at $9.46 \mu \mathrm{m}$ ), respectively. Note in Fig. 2(b) only the arbitrary unit is shown as the spectra have been lifted for clarity. The difference in the peak wavelength between the two polarizations is due to the $D_{2 d}$ tetragonal perturbation of the local crystal and the strain-field effects on the QW. ${ }^{9}$ The response peaks are redshifted with respect to the as-
Downloaded 13 Nov 2006 to 147.8.21.97. Redistribution subject to AIP license or copyright, see http://apl.aip.org/apl/copyright.jsp

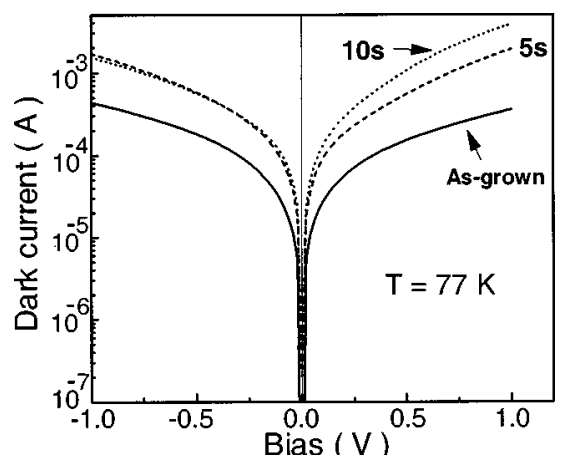

FIG. 3. $I-V$ curves of the as-grown, $5 \mathrm{~s}$, and $10 \mathrm{~s}$ annealed samples at $77 \mathrm{~K}$ as a function of bias.

The redshift of the interdiffused absorption peaks in Fig. 2 may indicate that the bound- and excited-state energies are being modified, and/or that the interdiffusion-induced changes in the depolarization shift, ${ }^{4}$ resulting in the postgrowth tuning of the absorption wavelength. The annealed absorption spectra at $0^{\circ}$ polarization decrease in amplitude and undergo broadening when compared to the as-grown spectrum, though the linewidth and amplitude of the two annealed spectra do not differ much, except for the continuing wavelength shift. The outdiffusion of the $\mathrm{Si}$ dopant across the heterointerfaces during the intermixing process not only reduces the free-carrier concentration, but also enhances the layer intermixing. ${ }^{10}$ Therefore, the broadening and the decrease in amplitude of the annealed absorption spectra may be attributed to the overall effect, which consists of reduction in carrier density, impurity scattering, and modification in the subband structure.

Leakage current was measured at $77 \mathrm{~K}$ using a 4156A parameter analyzer and cold finger. The $(I-V)$ characteristic is shown in Fig. 3. Note the asymmetry of the $I-V$ curves between the two polarities. For the as-grown samples, the leakage current is larger in reverse bias (i.e., mesa top negative) than in forward bias, which is attributed to dopant segregation during growth. ${ }^{11}$ But the reverse is true for the annealed devices, where the leakage currents are larger at forward bias, and they intersect and overlap with each other in reverse bias. This is due to the difference in In and $\mathrm{Ga}$ diffusion rates across the interfaces, which results in an asymmetric barrier height ${ }^{12}$ for the thermally excited electrons, and the redistribution of dopant impurity as described above. A direct consequence of these is the introduction of defects into the barrier that enhances the mechanism of defect-assisted tunneling. ${ }^{13}$ Since dark current is generally a thermionic emission in nature at $T>50 \mathrm{~K}$, these two factors, together with the thinner $300 \AA$ barrier and possibly the strain-induced tunneling, are believed to have resulted in the asymmetric $I-V$ curves and an increase in leakage current by nearly an order of magnitude at $77 \mathrm{~K}$, as a consequence of annealing.

The responsivity spectra were measured using a grating monochromator and glowbar source with lock-in detection and a $45^{\circ}$ polished facet. The polarizer was inserted before the glowbar source to study the polarization dependence of the photoresponse. Figure 4 shows the response spectra at $0^{\circ}$ and $90^{\circ}$ polarizations as a function of wavelength at $25 \mathrm{~K}$. 


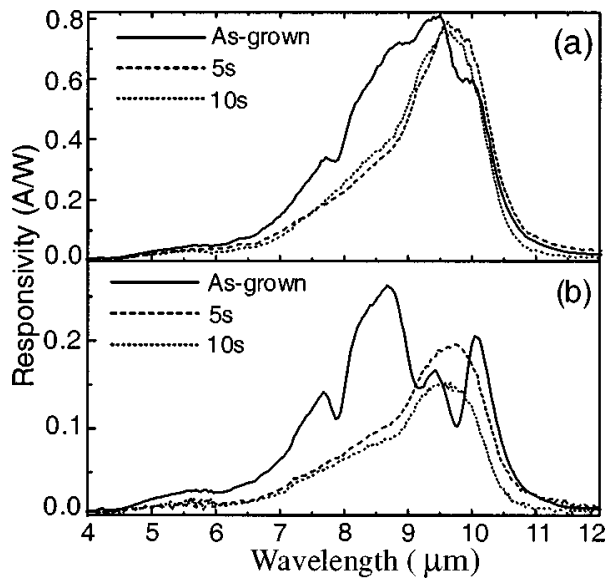

FIG. 4. Photoresponse spectra at $25 \mathrm{~K}$ of the as-grown (at $2.5 \mathrm{~V}$ ), $5 \mathrm{~s}$ (at $1.05 \mathrm{~V}$ ), and $10 \mathrm{~s}$ (at $1.65 \mathrm{~V}$ ) annealed samples at (a) $0^{\circ}$ polarization, and (b) $90^{\circ}$ polarization, as a function of wavelength.

grown spectrum at both polarizations. However, unlike the annealed absorption spectra, the peak positions of the annealed photoresponse do not differ significantly at both polarizations. This phenomenon is probably due to the results of different extents of intermixing on the band structure, polarization effect, low-temperature influence, and difference in applied bias. Further work is required to clarify this phenomenon.

Another feature worth noting is the satellite peaks that occur at a rather identical wavelength position in both asgrown spectra. As the polarization angle is changed from $0^{\circ}$ to $90^{\circ}$, four pronounced response peaks are observed in the as-grown spectrum of Fig. 4(b), with the dominant peak response occurring at a shorter, but not the designed, wavelength. Since there is only one bound state in the well and the excited states are above the barrier in the continuum, these satellite peaks are most probably due to the intersubband transition from the bound state $E_{1}$ to other excited states in the continuum, ${ }^{14}$ or the interaction between the excited state $E_{2}$ and other states in the continuum. ${ }^{15}$ However, with the modification of the QW structure by interdiffusion, the annealed spectra in Fig. 4 show that, regardless of polarization, all these satellite peaks are subdued, except the designed transition peak. Figure $4(\mathrm{a})$ shows that at $0^{\circ}$ polarization the corresponding responsivity amplitudes $(0.8,0.79$, and 0.77 $\mathrm{A} / \mathrm{W})$ do not vary much for the as-grown and annealed $\left(t_{A}\right.$ $=5$ and $10 \mathrm{~s}$ ) detectors. Note also that the three spectra in Fig. 4(a) have rather identical cutoff wavelengths. This is expected, since the MQW structure has not been substantially modified after interdiffusion; once the electrons are being photoexcited, they are ready to be collected as the photocurrent. While at $90^{\circ}$ polarization, Fig. 4(b) shows that the photoresponse spectra exhibit different features, and the $5 \mathrm{~s}$ annealed peak response is enhanced in amplitude in the wavelength range of interest between 9 and $10 \mu \mathrm{m}$.

In conclusion, the postgrowth tunability of a high In composition, pseudomorphic InGaAs/GaAs QWIP using $\mathrm{SiO}_{2}$-capped dopant-enhanced interdiffusion has been demonstrated. PL measurements show that no catastrophic deterioration in the MQW structures is observed. The TE polarization infrared intersubband transition, as a consequence of the band-mixing effects, is preserved. Both the $0^{\circ}$ and $90^{\circ}$ polarization absorption peaks are redshifted with respect to the as-grown one without much degradation in absorption strength. Photoresponse peaks due to resonances in the continuum states are subdued after interdiffusion. The annealed response spectra at $0^{\circ}$ polarization are comparable to the as-grown device with narrower FWHM, and the designed photoresponse peak becomes dominant at $90^{\circ}$ polarization following RTA. Intermixing will introduce defects (Ga vacancy) and dopant impurities into the barriers. These will assist electron tunneling and may possibly increase the density of dislocation-related point defects, thereby causing annealed dark currents to be about an order higher in amplitude than the as-grown one at $77 \mathrm{~K}$. By optimizing the QWIP structure to impede the tunneling mechanism, a high detectivity interdiffusion tunable photodetector is achievable for broadband and multicolor IR applications. However, a complete understanding of the effect of heat treatment on strained layers requires much more work.

This work is supported in part by the HKU-CRCG, RGC-Earmarked Research Grants and Academic Research Fund of the National University of Singapore. The authors would like to thank Professor S. J. Chua for valuable suggestions, as well as T. Mei and Dr. S. J. Xu for technical assistance.

${ }^{1}$ K. K. Choi, The Physics of Quantum Well Infrared Photodetectors (World Scientific, Singapore, 1997).

${ }^{2}$ R. P. G. Karunasiri, J. S. Park, J. Chen, R. Shih, J. F. Schdihing, and M. A. Dodd, Appl. Phys. Lett. 67, 2600 (1995).

${ }^{3}$ Quantum Well Mixing and Optoelectronic Device Applications, Milestone Series, Vol. 145, edited by E. H. Li (SPIE, Bellingham, WA, 1998).

${ }^{4}$ J. D. Ralston, M. Ramsteiner, B. Discher, M. Maier, G. Brandt, P. Koidl, and D. J. As, J. Appl. Phys. 70, 2195 (1991).

${ }^{5}$ A. G. Steele, M. Buchanan, H. C. Liu, and Z. R. Wasilewski, J. Appl. Phys. 75, 8234 (1994).

${ }^{6}$ P. L. Gourley, I. J. Fritz, and L. R. Dawson, Appl. Phys. Lett. 52, 377 (1988).

${ }^{7}$ S. Bürker, M. Baeumler, J. Wanger, E. C. Larkins, W. Rothemund, and J. D. Ralston, J. Appl. Phys. 79, 6818 (1996).

${ }^{8}$ L. H. Peng and C. G. Fonstad, J. Appl. Phys. 77, 747 (1995).

${ }^{9}$ L. H. Peng, J. H. Smet, T. P. E. Broekaert, and C. G. Fonstad, Appl. Phys. Lett. 61, 2078 (1992).

${ }^{10}$ D. G. Deppe and N. Holonyak, Jr., J. Appl. Phys. 64, R93 (1988).

${ }^{11}$ H. C. Liu, Z. R. Wasilewski, M. Buchanan, and H. Y. Chu, Appl. Phys. Lett. 63, 761 (1993).

${ }^{12}$ A. S. W. Lee and E. H. Li, Appl. Phys. Lett. 69, 3581 (1996).

${ }^{13}$ G. M. William, R. E. DeWames, C. W. Farley, and R. J. Anderson, Appl. Phys. Lett. 62, 1324 (1992).

${ }^{14}$ K. M. S. V. Bandara, B. F. Levine, and M. T. Asom, J. Appl. Phys. 74, 346 (1993).

${ }^{15}$ K. K Choi, M. Taysing-Lara, P. G. Newman, and W. Chang, Appl. Phys. Lett. 61, 1781 (1992). 